\title{
Erratum to: Spectrum and frequencies of BRCA1/2 mutations in Bulgarian high risk breast cancer patients
}

Rumyana Ivanova Dodova ${ }^{1,2+}$, Atanaska Velichkova Mitkova ${ }^{1,2^{*}}$, Daniela Rosenova Dacheva ${ }^{1,2}$, Lina Basam Hadjo , Alexandrina Ivanova Vlahova, ${ }^{3,4}$, Margarita Stoyanova Taushanova - Hadjieva ${ }^{5}$, Spartak Stoyanov Valev, Marija Mitko Caulevska', Stanislava Dimitrova Popova' ${ }^{1}$, Ivan Emilov Popov ${ }^{1,2}$, Tihomir lliichev Dikov,4, Theophil Angelov Sedloev 6,7, Atanas Stefanov lonkov ${ }^{8,9}, K^{6}$ stanta Velinova Timcheva ${ }^{5}$, Svetlana Liubomirova Christova ${ }^{3,4}$, Ivo Marinov Kremensky ${ }^{1}$, Vanio Ivanov Mitev ${ }^{1,2}$ and Radka Petrova Kaneva ${ }^{1,2}$

\section{Erratum}

In this version of this article that was originally published [1] there was an error with the citation in the line: "...Functional studies suggested that c.536A > G but not c.1648A > $\mathrm{C}$ variant might be related to BRCA1-associated pathogenicity by affecting its function in non-homologous end joining (NHEJ) [25]..."

The reference number 25 is incorrect for this citation. The correct reference does not appear in the article, but should be:

Guidugli L, Rugani C, Lombardi G, Aretini P, Galli A, Caligo MA. A recombination-based method to characterize human BRCA1 missense variants. Breast Cancer Red Treat. 2011 125(1):265-72.

\section{Author details}

${ }^{1}$ Molecular Medicine Center, Medical University of Sofia, 2 Zdrave str, 1431 Sofia, Bulgaria. ${ }^{2}$ Department of Medical Chemistry and Biochemistry, Medical Faculty, Medical University of Sofia, 2 Zdrave str, 1431 Sofia, Bulgaria. ${ }^{3}$ General and Clinical Pathology Clinic, University Hospital "Alexandrovska", 1 Georgi Sofiiski str, 1431 Sofia, Bulgaria. ${ }^{4}$ Department of General and Clinical Pathology, Medical University of Sofia, 1 Georgi Sofiiski str, 1431 Sofia, Bulgaria. ${ }^{5}$ Clinic of Medical Oncology (Chemotherapy), Specialized Hospital for Active Treatment in Oncology, 6 "Plovdivsko pole" str, 1756 Sofia, Bulgaria. "Department of Surgery, University Hospital "Tsaritsa Yoana - ISUL", 8 "Byalo more" str, 1527 Sofia, Bulgaria. "Medical Faculty, 8 "Byalo more" str, 1527 Sofia, Bulgaria. ${ }^{8}$ Department of General and Liver-Pancreatic Surgery, University Hospital "Alexandrovska", 1 Georgi Sofiiski str, 1431 Sofia, Bulgaria. ${ }^{9}$ Medical Faculty, Medical University of Sofia, 1 Georgi Sofiiski str, 1431 Sofia, Bulgaria.
Received: 10 March 2017 Accepted: 10 March 2017

Published online: 16 March 2017

\section{Reference}

1. Dodova RI, Mitkova AV, Dacheva DR, Hadjo LB, Vlahova Al, TaushanovaHadjieva MS, et al. Spectrum and frequencies of BRCA1/2 mutations in Bulgarian high risk breast cancer patients. BMC Cancer. 2015;15:523.

\footnotetext{
* Correspondence: mitkova@mmcbg.org

${ }^{\dagger}$ Equal contributors

${ }^{1}$ Molecular Medicine Center, Medical University of Sofia, 2 Zdrave str, 1431

Sofia, Bulgaria

2Department of Medical Chemistry and Biochemistry, Medical Faculty,

Medical University of Sofia, 2 Zdrave str, 1431 Sofia, Bulgaria

Full list of author information is available at the end of the article
} 